\title{
Butterflies of Athgarh Forest Division, Odisha, Eastern India, with notes on some significant records
}

\author{
Arajush Payra' ${ }^{1}$, Suraj K. Dash ${ }^{1}$, Udit P. Das ${ }^{2}$, Himanshu S. Palei ${ }^{3 *}$, Arun K. Mishra ${ }^{4}$ \\ ${ }^{1}$ Department of Wildlife and Biodiversity Conservation, North Orissa University, Odisha, India \\ ${ }^{2}$ Department of Zoology, North Orissa University, Odisha, India \\ ${ }^{3}$ Aranya Foundation, 625/12, Mars Villa, Panchasakha Nagar, Bhubaneswar, Odisha, India \\ ${ }^{4}$ Divisional Forest Office, Athgarh Forest Division, Cuttack, Odisha, India \\ ${ }^{*}$ Corresponding author E-mail: arajushpayra@gmail.com
}

The present paper deals with first annotated list of butterflies from Athgarh Forest Division, Odisha, India. A total of 136 species belongs to six families were recorded during January 2015 to September 2015. Notes on some of the significant record of butterflies for the region, were provided along with their distribution. Among the recorded 136 species of butterflies, 14 species are legally protected under Indian Wildlife Protection Act, 1972.

Key words: Balikiari Reserve Forest; Tersing; butterfly; checklist; distribution

\section{Introduction}

Butterflies occupy vital position in natural ecosystem as their adults are very good pollinators and larvae act as primary herbivores (Choudhury et al., 2012). They directly depend on plants for their entire life span from larval host plant to nectar of flowers and their diversity may serve as a surrogate for plant diversity (Harisha \& Hosetti, 2013). Due to their attractive coloured wings and fluttering flight, butterflies always attract the attention of naturalists, researchers, and conservationists. There are about 1501 species of butterflies in Indian subcontinent (Kunte et al., 1999), out of which 150 species have been recorded from Eastern Ghats (Gunathilagaraj et al., 1998), 334 species from Western Ghats (Tiple et al., 2009) and 962 species from North East region (Evans, 1932). In Odisha, first faunistic study on butterfly fauna presented by Taylor \& de Niceville (1888). They reported a list of butterflies from Khurda district. Later, Crawford (1921) noted some butterflies from Meghasani hills of Mayurbhanj district and at the same time, Annandale \& Dover (1921) published a list on butterflies from the Barkuda Island of Chilka. Afterwards, many noticeable woks on butterflies have been carried out by several workers in different parts of the state (Mandal \& Nandi 1984; Mandal \& Moulik 1991; Sahu et al. 2006; Sethy et al. 2006; Sethy \& Jana 2009; Nair, 2007, 2011; Das \& Sahu 2011; Mohapatra et al. 2012; Palei \& Rath, 2014; Payra et al. 2016; Paria et al. 2018; Boruah et al. 2019). As there is no literature available on butterfly diversity in Athgarh Forest Division, an attempt was made to strengthen the information on diversity and distribution of butterflies in Odisha through this present study.

\section{Materials and Methods}

Athgarh Forest Division situated in Cuttack district comprises of five Ranges i.e. Athgarh, Baramba, Khuntuni, Narasinghpur East and Narasinghpur West over an area of $1510 \mathrm{Km}^{2}$ (Fig. 1). It is located between latitude 20021'19.2" $\mathrm{N}$ to $20^{\circ} 40^{\prime} 27.6^{\prime \prime} \mathrm{N}$ and longitude 85052'0.72" E to 84'55'42.8"E. There are 37 Reserve Forests in Athgarh Division. This division is surrounded by Satkosia Tiger Reserve in the West, Cuttack Forest Division and City Forest Division in the East, Chandaka Wild Life Sanctuary, Nayagarh Forest Division and some part of Mahanadi Wildlife Division in the South and Dhenkanal Forest Division in the North. In Athgarh Forest Division, forest types are mainly Peninsular Sal forest, Dry Mixed-deciduous Forest, small patches of Semi-evergreen forest and Scrub forest (Champion \& Seth, 1968). The climate condition of the area experiences three distinct seasons: monsoon (July to September), winter (October to February) and summer (March to June). The area receives an average annual rainfall of $1400 \mathrm{~mm}$ to $1500 \mathrm{~mm}$ and the temperature ranges from $9^{\circ} \mathrm{C}$ in winter to $42^{\circ} \mathrm{C}$ in summer. 


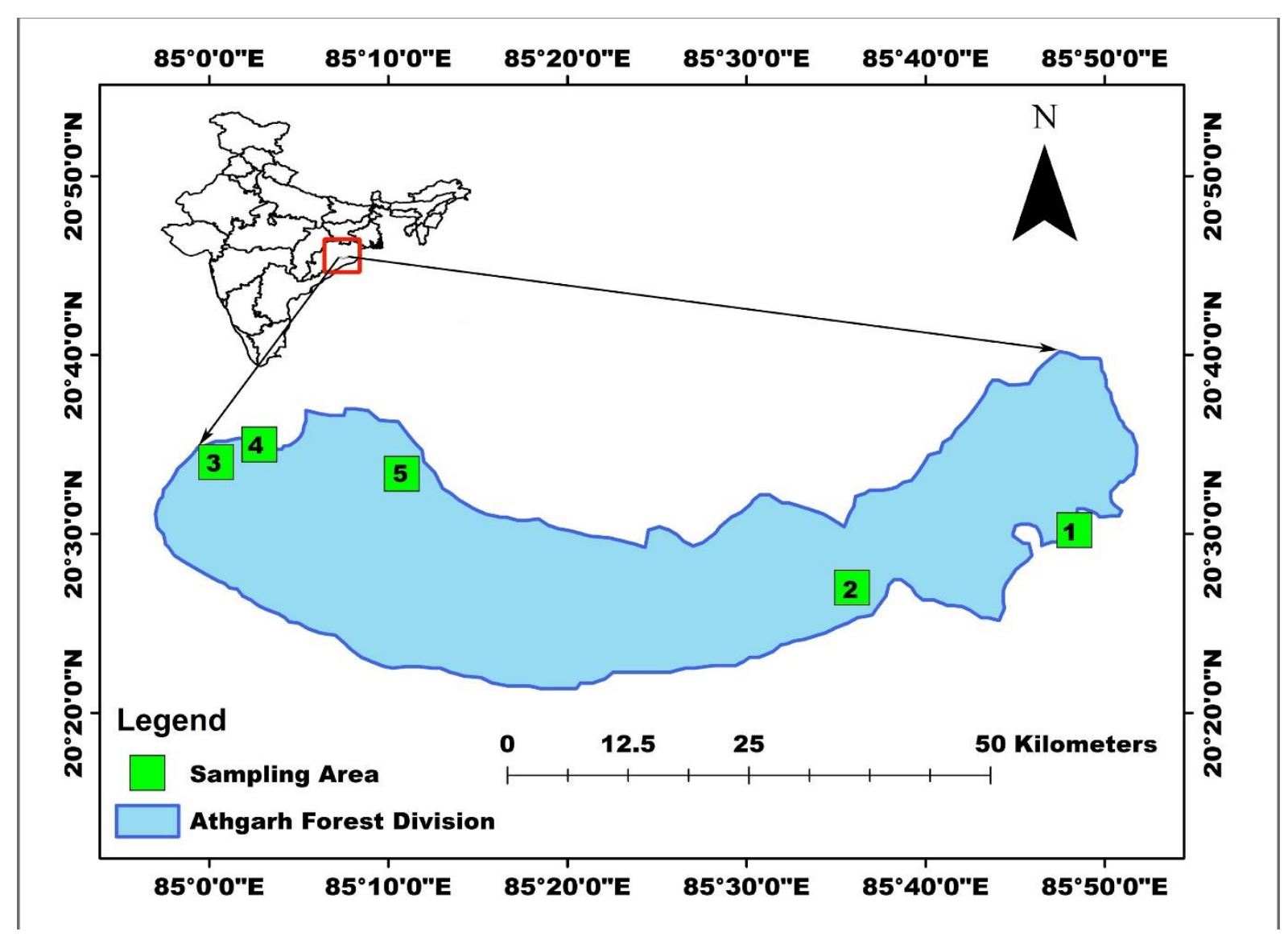

Fig. 1. Location map of study sites in Athgarh Forest Division, Odisha, Eastern India

Five sites were surveyed for the documentation of butterflies in Athgarh Forest Division.

Site 1 - Dhabaleswar Island $\left(20.503^{\circ} \mathrm{N}, 85.805^{\circ} \mathrm{E}, 19 \mathrm{~m}\right.$ a.s.l). It is a small island on Mahanadi River, comes under Khuntuni Range of Athgarh Forest Division. The frequently found plant species of this area are Ficus bengalensis, Ficus religiosa, Cassia siamea, Sizigium cumuni, Polyalthia longifolia, Azadirachta indica etc. Besides the above, the composites of the shrub forest are Lantana camara, Sida spinosa, Justicia adhatoda, Calotropis gigantea etc.

Site 2 - Ansupa Lake $\left(20.459^{\circ} \mathrm{N}, 85.602^{\circ} \mathrm{E}, 30 \mathrm{~m}\right.$ a.s.l). Ansupa lake is one of the largest freshwater lakes of Odisha spreading over 140 ha, situated at the left bank of Mahanadi River. It is surrounded by undulating plain and isolated hill ranges. The surrounding vegetation of the lake is mainly characterized by Cashew plantation, Agricultural land, patches of bamboo and hilly thorny scrub forest.

Site 3 - Balikiari Reserve Forest $\left(20.552^{\circ} \mathrm{N}, 85.038^{\circ} \mathrm{E}, 233 \mathrm{~m}\right.$ a.s.l.). With an area of 5217.87 hectare, this Reserve Forest (RF) is situated in Narsingpur West Range. Small streams flow within this RF mainly in rainy season, but mostly remain dry for rest of the seasons. Mangifera indica, Shorea robusta, Aegle marmelos, Anogeissus latifolia, Dalbergia latifolia, Diospyros sp, Azadirachta indica are dominated floral components of this region.

Site 4 - Tersing $\left(20.582^{\circ} \mathrm{N}, 85.047^{\circ} \mathrm{E}, 435 \mathrm{~m}\right.$ a.s.l.). This is the bordering area between Athgarh Forest Division and Satkosia Tiger Reserve. It lies at an altitude of about $450 \mathrm{~m}$. The dominant trees are mainly Aeg/e marmelos, Terminalia tomentosa, Shorea robusta, Ficus religiosa, Diosspyros melanoxylon, Syzigium cumini, Cicus sp etc.

Site 5 - Deobhuin Reserve Forest $\left(20.556^{\circ} \mathrm{N}, 85.179^{\circ} \mathrm{E}, 160 \mathrm{~m}\right.$ a.s.I.). This reserve forest is situated in Narsingpur East Range with an area of $6082.23 \mathrm{ha}$. Slow flowing streams, and one waterfall is present within this reserve forest. The vegetation is dominated by Shorea robusta, Xilia xylocarpa, Terminalia tomentosa, Schleichera oleosa, Lagerstroemia parviflora etc.

\section{Data Collection and Identification}

Butterflies were observed through opportunistic survey from January 2015 to September 2015. During this inventory all possible and suitable habitats of butterflies, like agricultural land, forest trails, streams, waterfall, nectaring plants were surveyed. All observations were mainly carried out between $7.00 \mathrm{am}$ to $11.00 \mathrm{am}$ and $2.30 \mathrm{pm}$ to $4.30 \mathrm{pm}$. Most of the species were photographed in the field by using Nikon D3200 Camera with Tamron 70-300 mm lens. Coordinates and elevations of study sites were obtained by using Garmine eTrex and Google earth. An entomological net was also used for capturing butterflies, which were released immediately at the spot of capture without any harm. Butterflies were identified using available literature of Evans (1932, 1949), and photographic guidebooks of Haribal (1992) and Kehimkar (2008, 2016) together with website of Indian butterflies (https://www.ifoundbutterflies.org/). Scientific names and common names presented according to Kunte et al. (2019), Varshney \& Smetacek, (2015). 


\section{Results}

A total of 136 species of butterflies belonging to six families were recorded in the study area (See Table 1). The most dominant family was Lycaenidae with 41 species (30.15\%), followed by Nymphalidae (28.68\%, 39 species), Hesperiidae (19.85\%, 27 species), Pieridae (11.76\%, 16 species), Papilionidae (8.82\%, 12 species) and Riodinidae (1 species, 0.74\%). Notes on some of the significant records of butterflies were provided below along with their distribution.

Table 1. List of the recorded butterflies from Athgarh Forest Division, Odisha, Eastern India

\begin{tabular}{|c|c|c|c|c|c|c|c|c|}
\hline \multirow[t]{2}{*}{ SI.No } & \multirow[t]{2}{*}{ Family / Scientific Name } & \multirow[t]{2}{*}{ Common Name } & \multicolumn{5}{|c|}{ Study Sites } & \multirow{2}{*}{$\begin{array}{l}\text { Figure } \\
\text { number }\end{array}$} \\
\hline & & & S1 & S2 & S3 & S4 & S5 & \\
\hline \multicolumn{9}{|c|}{ Family Hesperiidae } \\
\hline 1 & Hasora chromus (Cramer, [1780]) & $\begin{array}{l}\text { Common Banded } \\
\text { Awl }\end{array}$ & & + & + & + & & $2 a$ \\
\hline 2 & Hasora vitta (Butler, 1870) & Plain Banded Awl & & & & & + & 20 \\
\hline 3 & $\begin{array}{l}\text { Badamia exclamationis (Fabricius, } \\
\text { 1775) }\end{array}$ & Brown Awl & & + & + & & + & $2 b$ \\
\hline 4 & $\begin{array}{l}\text { Celaenorrhinus leucocera (Kollar, } \\
\text { [1844]) }\end{array}$ & $\begin{array}{l}\text { Common Spotted } \\
\text { Flat }\end{array}$ & & & + & & & \\
\hline 5 & Coladenia indrani(Moore, [1866]) & Tricolour Pied Flat & & & + & + & + & $2 c$ \\
\hline 6 & Tagiades litigiosa Möschler, 1878 & Water Snow Flat & & & + & + & + & $2 d$ \\
\hline 7 & Tagiades japetus (Stoll, [1781]) & Common Snow Flat & + & + & + & + & + & \\
\hline 8 & Caprona ransonnettii (R. Felder, 1868) & Golden Angle & & + & + & + & + & $2 \mathrm{e}$ \\
\hline 9 & Sarangesa dasahara (Moore, [1866]) & Common Small Flat & & + & + & + & + & $2 f$ \\
\hline 10 & Spialia galba (Fabricius, 1793) & Indian Skipper & + & + & & & & \\
\hline 11 & Ampittia dioscorides (Fabricius, 1793) & Bush Hopper & & + & + & & + & $2 g$ \\
\hline 12 & lambrix salsala (Moore, [1866]) & Chestnut Bob & + & + & + & + & + & \\
\hline 13 & Suastus gremius (Fabricius, 1798) & Indian Palm Bob & + & + & + & & + & \\
\hline 14 & Zographetus satwa de Nicéville, 1884 & $\begin{array}{l}\text { Purple-and-gold } \\
\text { Flitter }\end{array}$ & & & + & & & $2 \mathrm{~h}$ \\
\hline 15 & Udaspes folus (Cramer, [1775]) & Grass Demon & + & & + & & + & $2 \mathrm{i}$ \\
\hline 16 & $\begin{array}{l}\text { Notocrypta curvifascia (C. \& R. Felder, } \\
\text { 1862) }\end{array}$ & Restricted Demon & & & & + & & $2 \mathrm{j}$ \\
\hline 17 & $\begin{array}{l}\text { Notocrypta paralysos (Wood-Mason \& } \\
\text { de Nicéville, 1881) }\end{array}$ & $\begin{array}{l}\text { Common Banded } \\
\text { Demon }\end{array}$ & & & & + & + & \\
\hline 18 & Hyarotis adrastus (Stoll, [1780]) & Tree Flitter & & + & & & & \\
\hline 19 & Matapa aria (Moore, [1866]) & Common Redeye & + & + & + & + & + & \\
\hline 20 & Oriens goloides (Moore, [1881]) & Ceylon Dartlet & & & + & + & + & \\
\hline 21 & Potanthus sp. & Dart & & & & + & & $2 k$ \\
\hline 22 & Telicota sp. & Palm Dart & & & + & + & + & \\
\hline 23 & Parnara sp. & Swift & + & + & + & & + & \\
\hline 24 & Pelopidas mathias (Fabricius, 1798) & Small Branded Swift & + & + & + & & + & $2 n$ \\
\hline 25 & Baoris farri(Moore, 1878) & Paint-brush Swift & & & + & + & + & 21 \\
\hline 26 & Halpe porus (Mabille, [1877]) & Moore's Ace & & & + & + & + & $2 \mathrm{~m}$ \\
\hline 27 & Caltoris sp. & Swift & & & + & & & \\
\hline \multicolumn{9}{|c|}{ Family Papilionidae } \\
\hline 28 & Graphium doson (C. \& R. Felder, 1864) & Common Jay & + & + & + & + & + & \\
\hline 29 & $\begin{array}{l}\text { Graphium agamemnon (Linnaeus, } \\
\text { 1758) }\end{array}$ & Tailed Jay & + & + & + & + & + & \\
\hline 30 & Graphium nomius (Esper, 1799) & Spot Swordtail & & & + & + & + & $3 a$ \\
\hline 31 & Graphium antiphates (Cramer, [1775]) & Five-bar Swordtail & & & & & + & \\
\hline 32 & Papilio polymnestor Cramer, [1775] & Blue Mormon & + & + & + & + & + & $3 b$ \\
\hline 33 & Papilio crino Fabricius, 1793 & $\begin{array}{l}\text { Common Banded } \\
\text { Peacock }\end{array}$ & + & + & + & + & + & $3 c$ \\
\hline 34 & Papilio nephelus Boisduval, 1836 & Yellow Helen & & & & + & + & \\
\hline 35 & Papilio polytes Linnaeus, 1758 & Common Mormon & + & + & + & + & + & $3 d$ \\
\hline 36 & Papilio clytia Linnaeus, 1758 & Common Mime & + & + & + & + & + & $3 f, 3 g$ \\
\hline 37 & Pachliopta hector(Linnaeus, 1758) & Crimson Rose & + & + & & & & $3 \mathrm{j}$ \\
\hline 38 & $\begin{array}{l}\text { Pachliopta aristolochiae (Fabricius, } \\
\text { 1775) }\end{array}$ & Common Rose & + & + & + & & + & $3 i$ \\
\hline
\end{tabular}




\begin{tabular}{|c|c|c|c|c|c|c|c|c|}
\hline 39 & Papilio demoleus Linnaeus, 1758 & Lime Butterfly & + & + & + & + & + & $3 \mathrm{~h}$ \\
\hline \multicolumn{9}{|c|}{ Family Pieridae } \\
\hline 40 & Belenois aurota Fabricius, 1793 & Pioneer & + & + & & & & $4 a$ \\
\hline 41 & Cepora nerissa Fabricius, 1775 & Common Gull & + & + & + & + & + & $4 \mathrm{~b}$ \\
\hline 42 & Delias eucharis Drury, 1773 & Common Jezebel & + & + & + & + & + & \\
\hline 43 & Delias hyparete Linnaeus, 1758 & Painted Jezebel & & + & & & & $4 c$ \\
\hline 44 & Appias olferna Swinhoe, 1890 & $\begin{array}{l}\text { Eastern Striped } \\
\text { Albatross }\end{array}$ & + & + & + & & + & \\
\hline 45 & Leptosia nina Fabricius, 1793 & Psyche & + & + & + & + & + & \\
\hline 46 & Ixias marianne(Cramer, [1779]) & White Orange-tip & & + & & & & $4 d$ \\
\hline 47 & Ixias pyrene (Linnaeus, 1764) & Yellow Orange-tip & + & + & + & & & \\
\hline 48 & Pareronia hippia Fabricius, 1787 & Common Wanderer & + & + & + & + & + & $4 \mathrm{e}$ \\
\hline 49 & Catopsilia pomona Fabricius, 1775 & Lemon Emigrant & + & + & + & + & + & $4 f$ \\
\hline 50 & Catopsilia pyranthe Linnaeus, 1758 & Mottled Emigrant & + & + & + & + & + & \\
\hline 51 & Eurema brigitta Stoll, 1780 & Small Grass Yellow & + & + & + & + & + & \\
\hline 52 & Eurema laeta Boisduval, 1836 & $\begin{array}{l}\text { Spotless Grass } \\
\text { Yellow }\end{array}$ & & & + & + & + & \\
\hline 53 & Eurema blanda Boisduval, 1836 & $\begin{array}{l}\text { Threespot Grass } \\
\text { Yellow }\end{array}$ & & + & + & + & + & $4 i$ \\
\hline 54 & Eurema hecabe Linnaeus, 1758 & $\begin{array}{l}\text { Common Grass } \\
\text { Yellow }\end{array}$ & + & + & + & + & + & $4 g$ \\
\hline 55 & Eurema andersonii (Moore, 1886) & $\begin{array}{l}\text { Onespot Grass } \\
\text { Yellow }\end{array}$ & & & + & + & + & $4 \mathrm{~h}$ \\
\hline \multicolumn{9}{|c|}{ Family Riodinidae } \\
\hline 56 & Abisara bifasciata Moore, 1877 & Double-banded Judy & & & + & + & + & $5 a$ \\
\hline \multicolumn{9}{|c|}{ Family Lycaenidae } \\
\hline 57 & Spalgis epeus (Westwood, [1851]) & Apefly & + & + & & & & \\
\hline 58 & Curetis thetis (Drury, [1773]) & Indian Sunbeam & + & & + & + & + & \\
\hline 59 & Prosotas nora Felder, 1860 & Common Lineblue & & + & + & + & + & \\
\hline 60 & Prosotas dubiosa Semper, 1879 & Tailless Lineblue & & + & + & + & + & $5 c$ \\
\hline 61 & Petrelaea dana (de Nicéville, [1884]) & Dingy Lineblue & & & & & & $5 b$ \\
\hline 62 & Caleta decidia (Hewitson, 1876) & Angled Pierrot & & & + & + & + & $5 d$ \\
\hline 63 & Jamides bochus Stoll, 1782 & Dark Cerulean & + & + & + & + & + & $5 \mathrm{e}$ \\
\hline 64 & Jamides celeno Cramer, 1775 & Common Cerulean & + & + & + & + & + & \\
\hline 65 & Catochrysops Strabo (Fabricius, 1793) & Forgetmenot & + & + & + & & + & $5 f$ \\
\hline 66 & Lampides boeticus (Linnaeus, 1767) & Pea Blue & + & + & + & & + & \\
\hline 67 & Leptotes plinius (Fabricius, 1793) & Zebra Blue & + & + & + & & + & $5 g$ \\
\hline 68 & Castalius rosimon (Fabricius, 1775) & Common Pierrot & + & + & + & + & + & \\
\hline 69 & Tarucus sp. & Pierrot & + & + & + & & + & $5 \mathrm{~h}$ \\
\hline 70 & Tarucus ananda (de Nicéville, [1884]) & Dark Pierrot & & & & + & & $5 i$ \\
\hline 71 & Zizeeria karsandra (Moore, 1865) & Dark Grass Blue & + & + & + & & + & \\
\hline 72 & Pseudozizeeria maha Kollar, 1844 & Pale Grass Blue & + & + & + & + & + & \\
\hline 73 & Zizina otis Fabricius, 1787 & Lesser Grass Blue & + & + & + & + & + & \\
\hline 74 & Zizula hylax (Fabricius, 1775) & Tiny Grass Blue & + & + & & & & \\
\hline 75 & Everes lacturnus Godart, 1824 & Indian Cupid & & & + & + & + & $5 j$ \\
\hline 76 & Neopithecops zalmora Butler, 1870 & Quaker & + & + & + & + & + & \\
\hline 77 & Megisba malaya (Horsfield, [1828]) & Malayan & & & + & + & + & $5 \mathrm{k}$ \\
\hline 78 & Acytolepis puspa (Horsfield, [1828]) & Common Hedge Blue & & & + & + & + & 51 \\
\hline 79 & Euchrysops cnejus (Fabricius, 1798) & Gram Blue & + & + & + & & + & \\
\hline 80 & Chilades lajus (Stoll, [1780]) & Lime Blue & + & + & + & + & + & \\
\hline 81 & Chilades pandava (Horsfield, [1829]) & Plains Cupid & + & + & + & + & + & \\
\hline 82 & Chilades parrhasius Fabricius, 1793 & Small Cupid & & & + & & & $5 m$ \\
\hline 83 & Freyeria putli (Kollar, [1844]) & Grass Jewel & + & + & + & & + & \\
\hline 84 & Anthene emolus (Godart, [1824]) & Ciliate Blue & + & + & + & & + & \\
\hline 85 & Anthene lycaenina (R. Felder, 1868) & Pointed Ciliate Blue & + & & & & & \\
\hline 86 & Spindasis vulcanus (Fabricius, 1775) & Common Silverline & + & + & + & + & + & \\
\hline 87 & Spindasis syama (Horsfield, [1829]) & Club Silverline & & & + & & + & $5 n$ \\
\hline 88 & Spindasis lohita (Horsfield, [1829]) & $\begin{array}{l}\text { Long-banded } \\
\text { Silverline }\end{array}$ & & & + & & & 50 \\
\hline
\end{tabular}


Butterflies of Athgarh Forest Division

\begin{tabular}{|c|c|c|c|c|c|c|c|c|}
\hline 89 & Arhopala atrax (Hewitson, 1862) & Indian Oakblue & & + & + & + & + & \\
\hline 90 & Arhopala amantes Hewitson, 1862 & Large Oakblue & & & + & + & + & \\
\hline 91 & Amblypodia anita Hewitson, 1862 & Purple Leaf Blue & & + & + & + & + & \\
\hline 92 & Loxura atymnus Stoll, 1780 & Yamfly & + & + & + & + & + & \\
\hline 93 & Chliaria othona (Hewitson, 1865) & Orchid Tit & & & + & & & $5 p$ \\
\hline 94 & Virachola isocrates (Fabricius, 1793) & Common Guava Blue & + & & + & & & $5 q$ \\
\hline 95 & Rapala varuna Horsfield, 1829 & Indigo Flash & & + & + & & & $5 r$ \\
\hline 96 & Rapala manea Hewitson, 1863 & Slate Flash & + & + & + & & + & \\
\hline 97 & Rapala iarbus (Fabricius, 1787) & Indian Red Flash & + & & + & & & \\
\hline \multicolumn{9}{|c|}{ Family Nymphalidae } \\
\hline 98 & Parantica ag/ea Stoll, 1782 & Glassy Tiger & & + & + & & & $6 a$ \\
\hline 99 & Tirumala limniace Cramer, 1775 & Blue Tiger & + & + & + & + & + & \\
\hline 100 & Danaus genutia Cramer 1779 & Common Tiger & + & + & + & + & + & \\
\hline 101 & Danaus chrysippus Linnaeus, 1758 & Plain Tiger & + & + & + & + & + & \\
\hline 102 & Euploea core (Cramer, [1780]) & $\begin{array}{l}\text { Common Indian } \\
\text { Crow }\end{array}$ & + & + & + & + & + & $6 b$ \\
\hline 103 & Melanitis leda Linnaeus, 1758 & $\begin{array}{l}\text { Common Evening } \\
\text { Brown }\end{array}$ & + & + & + & + & + & \\
\hline 104 & $\begin{array}{l}\text { Elymnias hypermnestra } \\
\text { Linnaeus, } 1763\end{array}$ & Common Palmfly & + & + & + & + & + & \\
\hline 105 & Lethe europa (Fabricius, 1775) & Bamboo Treebrown & + & + & + & & & \\
\hline 106 & Lethe rohria Fabricius, 1787 & Common Treebrown & & & + & & & $6 c$ \\
\hline 107 & Mycalesis perseus Fabricius, 1775 & Common Bushbrown & + & + & + & + & + & $6 d$ \\
\hline 108 & Orsotriaena medus Fabricius, 1775 & Nigger & & & + & + & & \\
\hline 109 & Ypthima huebneri Kirby, 1871 & Common Fourring & + & + & + & + & + & \\
\hline 110 & Ariadne ariadne Linnaeus, 1763 & Angled Castor & + & + & + & + & + & \\
\hline 111 & Ariadne merione Cramer, 1777 & Common Castor & + & & & & + & \\
\hline 112 & Phalanta phalantha Drury, 1773 & Common Leopard & + & + & + & + & + & \\
\hline 113 & Vanessa cardui Linnaeus, 1758 & Painted Lady & + & + & & & & \\
\hline 114 & Junonia hierta Fabricius, 1798 & Yellow Pansy & + & + & + & & + & \\
\hline 115 & Junonia orithya Linnaeus, 1758 & Blue Pansy & + & + & + & & + & \\
\hline 116 & Junonia lemonias Linnaeus, 1758 & Lemon Pansy & + & + & + & + & + & \\
\hline 117 & Junonia almana Linnaeus, 1758 & Peacock Pansy & + & + & + & + & + & \\
\hline 118 & Junonia atlites Linnaeus, 1763 & Grey Pansy & + & + & + & + & + & \\
\hline 119 & Junonia iphita Cramer, 1779 & Chocolate Pansy & + & + & + & + & + & \\
\hline 120 & Kallima inachus Doyere, 1840 & Orange Oakleaf & & & + & + & + & \\
\hline 121 & $\begin{array}{l}\text { Hypolimnas misippus (Linnaeus, } \\
\text { 1764) }\end{array}$ & Danaid Eggfly & & + & & & & $6 e$ \\
\hline 122 & Hypolimnas bolina Linnaeus, 1758 & Great Eggfly & + & + & + & + & + & \\
\hline 123 & Cyrestis thyodamas Doyère, 1840 & Common Map & & & & + & + & \\
\hline 124 & Neptis hylas Linnaeus, 1758 & Common Sailer & + & + & + & + & + & \\
\hline 125 & Neptis sappho Pallas, 1771 & Pallas Sailer & + & & & + & + & $6 f, 6 g$ \\
\hline 126 & Pantoporia hordonia Stoll, 1790 & Common Lascar & & & + & + & + & $6 \mathrm{~h}$ \\
\hline 127 & Athyma perius Linnaeus, 1758 & Common Sergeant & & & + & + & + & \\
\hline 128 & Moduza procris Cramer, 1777 & Commander & + & + & + & + & + & \\
\hline 129 & Tanaecia lepidea Butler, 1868 & Grey Count & & + & + & + & + & $6 i$ \\
\hline 130 & Symphaedra nais (Forster, 1771) & Baronet & & & + & + & + & $6 \mathrm{j}$ \\
\hline 131 & Euthalia aconthea Cramer, 1777 & Baron & + & + & + & & + & $6 \mathrm{k}$ \\
\hline 132 & Euthalia lubentina Cramer, 1777 & Gaudy Baron & & & & & + & \\
\hline 133 & Polyura athamas (Drury, [1773]) & Common Nawab & & & + & + & + & 61 \\
\hline 134 & Charaxes solon Fabricius, 1793 & Black Rajah & & & + & & + & \\
\hline 135 & Charaxes bernardus Fabricius, 1793 & Tawny Rajah & & & + & & & \\
\hline 136 & Acraea terpsicore (Linnaeus, 1758) & Tawny Coster & + & + & + & + & + & \\
\hline
\end{tabular}

\section{Hasora vitta (Butler, 1870) - Plain Banded Awl (Hesperiidae) (Fig. 20)}

A single individual was photographed at 11:25 (here and below local time, UTC+05:30) on 04.10.2015 in Deobhuin Reserve forest. This hesprid butterfly was perching under the leaf, at about $2 \mathrm{~m}$ height from the ground, along the forest trail. This butterfly ranges from Sikkim to Northeast India; Maharashtra to Goa up to Kerala (Varshney \& Smetacek, 2015). From Odisha, previously it was recorded from single locality, Mundasaru in Kandhamal District by Vivek Sarkar on 08.06 .2013 
(Kunte, 2019). Most recently this species has been reported from Maredumill, and Jalatarangini area of the adjoining state, Andhra Pradesh (Goswami et al., 2018). Apart from the record of Vivek Sarkar from Kandhamal District, no such decisive record of this butterfly is available from Odisha.

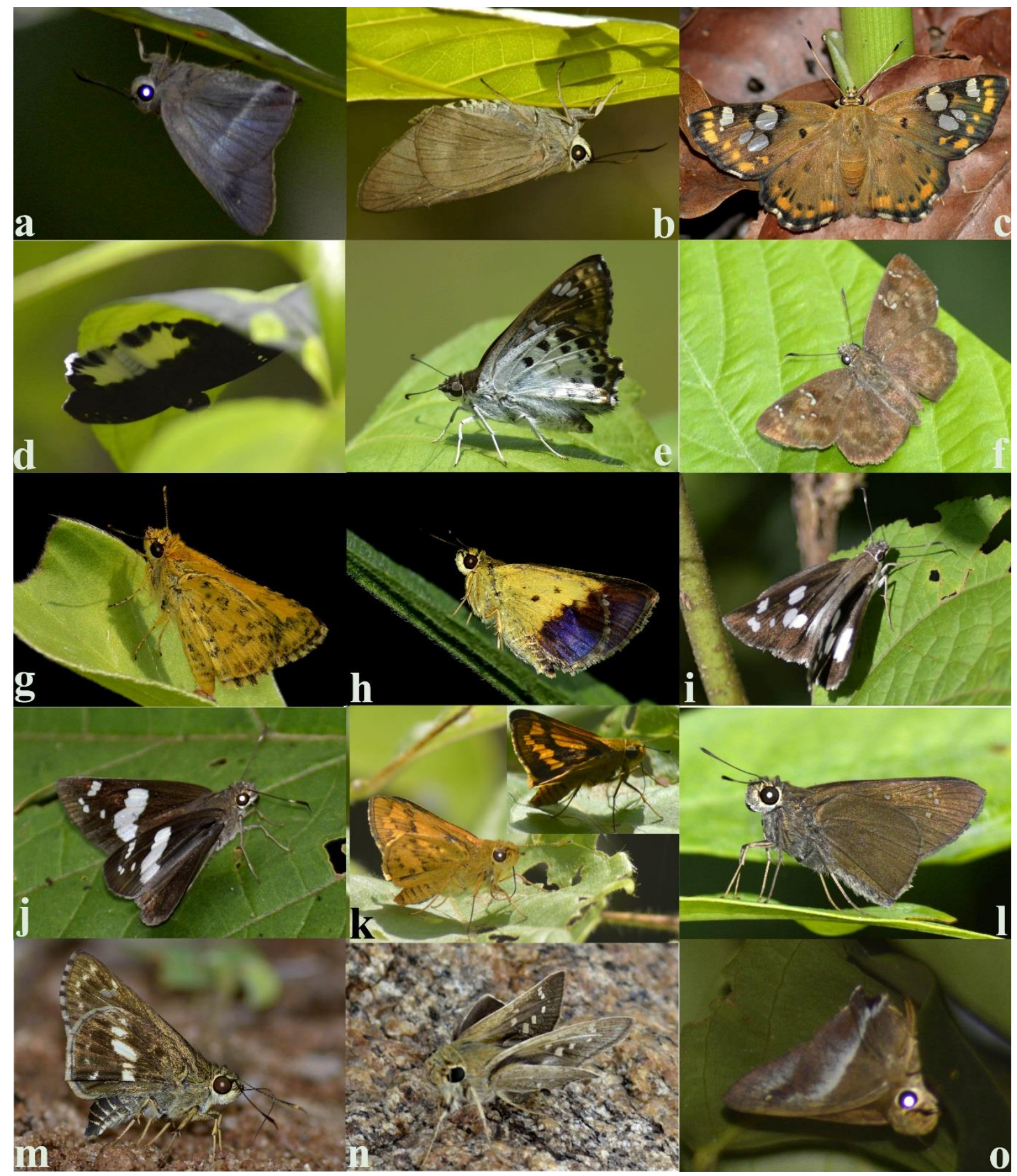

Fig. 2. Photographs of Hesperidae butterflies: a - Hasora chromus, b - Badamia exclamationis, c - Coladenia indrani, d Tagiades litigiosa Möschler, 1878; e - Caprona ransonnettii, f - Sarangesa dasahara; g - Ampittia dioscorides, h - Zographetus satwa; i - Udaspes folus, j - Notocrypta curvifascia; k - Potanthus sp.; I - Baoris farri, m - Halpe porus, $\mathrm{n}$ - Pelopidas mathias, o - Hasora vitta

\section{Zographetus satwa de Nicéville, 1884 - Purple and Gold Flitter (Hesperiidae) (Fig. 2h)}

We have recorded this species several times from different places of Balikiari Reserve Forest, during September 2015. On 01.09 we recorded two individuals. First, we spotted it at 11:50, near a stream. It was perching on shrubs at about $1 \mathrm{~m}$ above the ground. Later it was recorded during 15:05, at about $1 \mathrm{~km}$ distance from the first place of observation. It was perching on shrubs under dense canopy cover. On 02.09, we have encountered this species twice, Near the Sishupathra dam. This butterfly ranges from Uttarakhand to Northeast India (Varshney \& Smetacek, 2015). Previously, from Odisha only single record was available from same locality, i.e., Balikiari Reserve Forest, by Vivek Sarkar during September 2012 (Anonymous, 2019). 


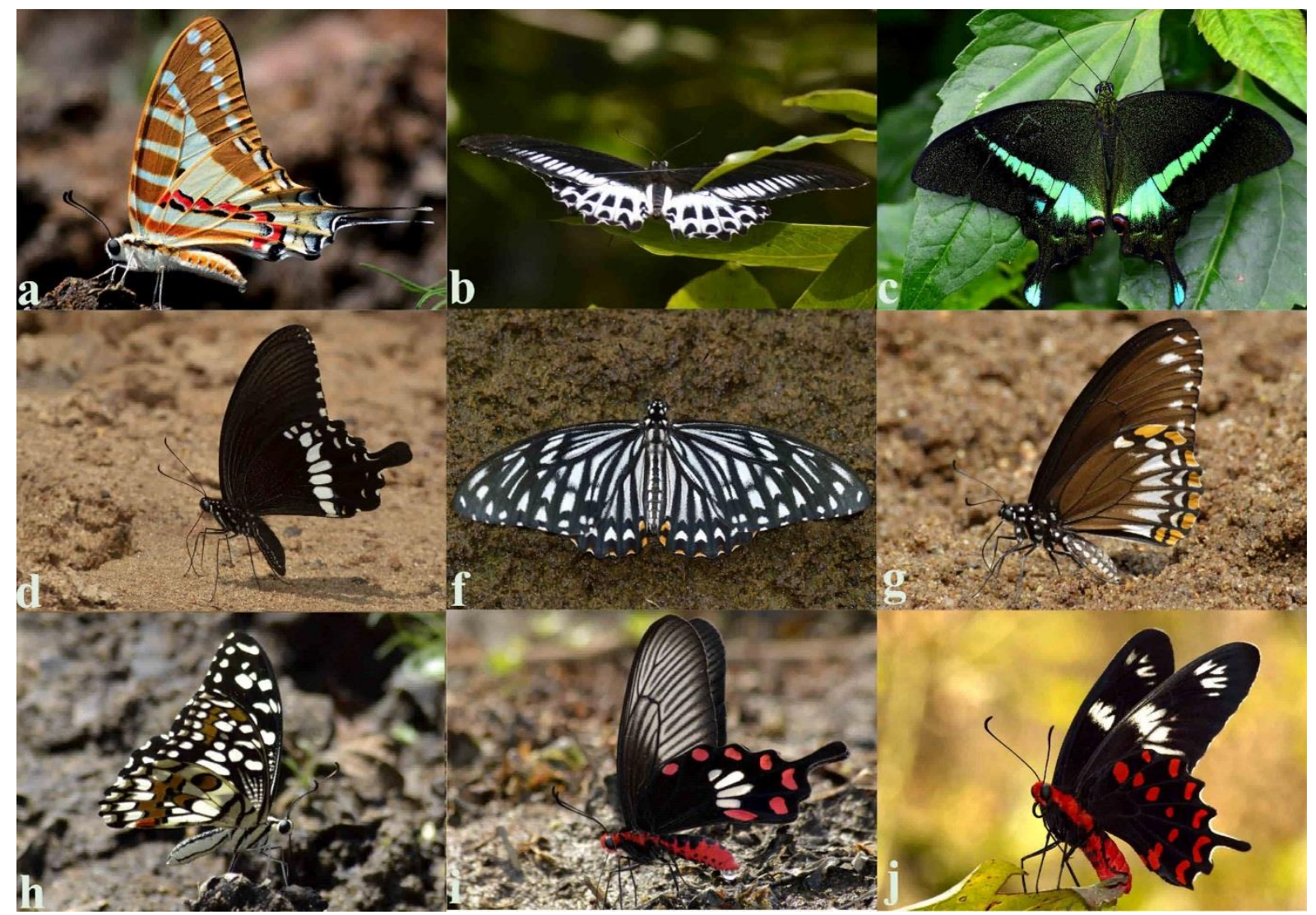

Fig 3. Photographs of Papilionidae butterflies: a - Graphium nomius, b - Papilio polymnestor, c - Papilio crino; $\mathrm{d}$ - Papilio polytes, f - Papilio clytia (form dissimilis); g - Papilio clytia (form clytia); h - Papilio demoleus, i - Pachliopta aristolochiae; j Pachliopta hector

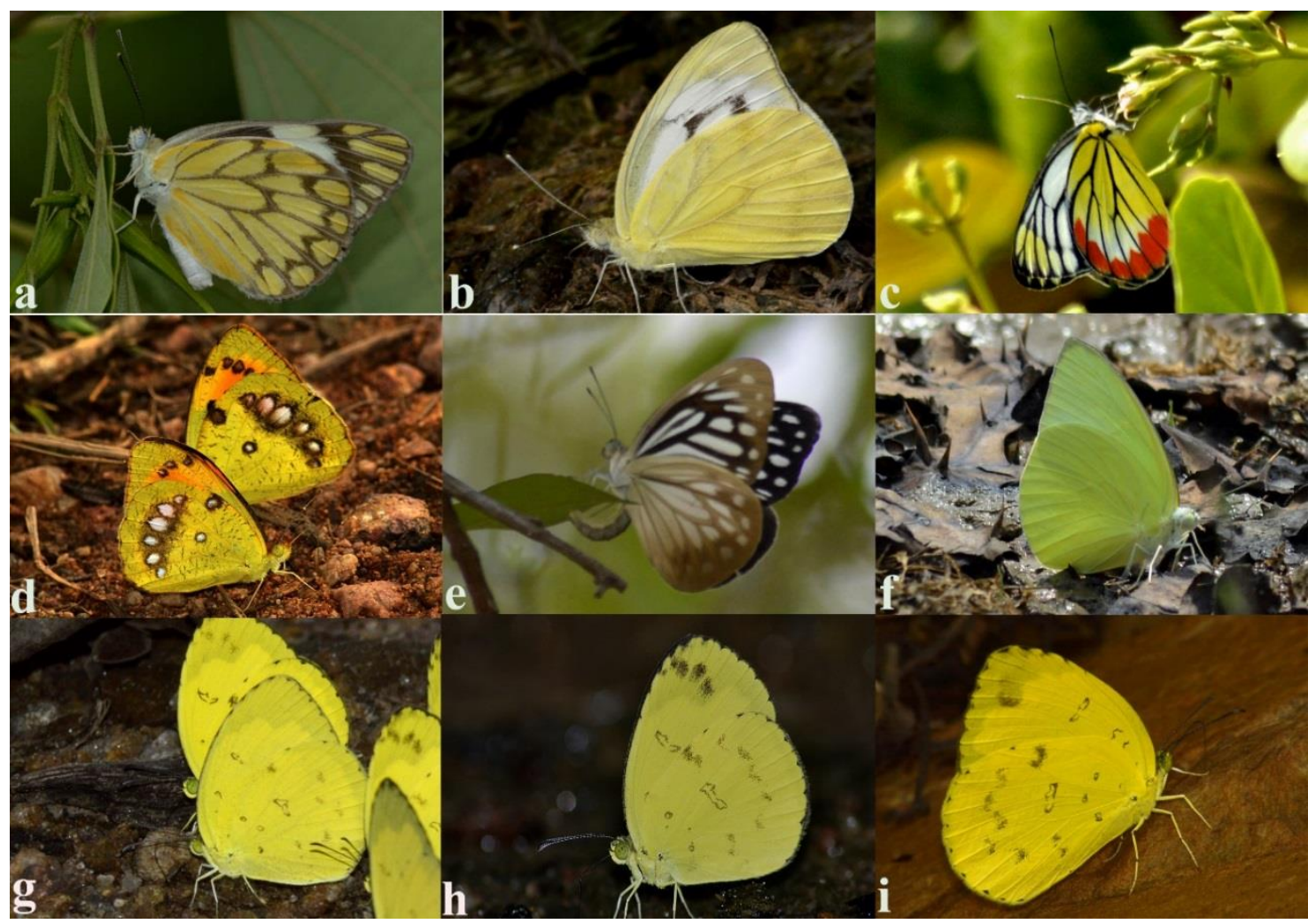

Fig 4. Photographs of Pieridae butterflies: a - Belenois aurota; b - Cepora nerissa; c - Delias hyparete; $\mathrm{d}$ - Ixias marianne; e Pareronia hippia; f - Catopsilia pomona; g - Eurema hecabe; h - Eurema andersonif; i Eurema blanda 
Potanthus sp. - Dart (Hesperiidae) (Fig. 2k)

One individual was photographed near Tersing on 03.09.2015 at 09:35 it was perching on the small shrubs, near the fastflowing hill stream. Until this date, no record is available of Potanthus $s p$. from Odisha. Recently the Genus has been reported from adjacent state Andhra Pradesh (Goswami et al., 2018). As Goswami et al. (2018) mentioned, without examination of genitalia species the identification is very difficult in this butterfly group. Further examination of specimen is required to validate the occurrence of this species in Odisha.

Tarucus ananda (de Nicéville, [1884]) - Dark Pierrot (Lycaenidae) (Fig. 5i)

On 03.09.2015, a single individual of Dark Pierrot Tarucus ananda was sighted from Tersing (Site 4) at 11:40. It was puddling on pond side sandy soil, along with other butterflies such as Common Mormon Papilio polytes, Angled Pierrot Caleta decidia and Common Nawab Polyura athamas. The distribution of this tiny butterfly is mainly from South-West India to North Maharastra, Sikkim to Arunachal Pradesh, Central Nepal, North East India and Burma to Dawnas (Evans, 1932; Kehimkar, 2008; Varshney \& Smetacek, 2015). In Odisha previously Vivek Sarkar recorded it during June 2013 from Balgaon Range of Khordha district (Churi, 2019). Our present record confirms its occurrence in Odisha.

Chliaria othona (Hewitson, 1865) - Orchid Tit (Lycaenidae) (Fig. 5p)

Single individual was recoded from Balikiari Reserve Forest, on 05.03.2015, at 11:35. It was puddling on sandy streambed. Place was covered by dense canopy cover. According to Varshney \& Smetacek (2015), this species ranges from Uttarakhand to Northeast India, Maharashtra to Karala. In Odisha previously Vivek Sarkar recorded it during June 2013 from Balgaon Range of Khordha district. Most recently, Subhajit Roy records it during June 2019 from Similipal National Park of Mayurbhanj District (Ogale et al., 2019).

Neptis sappho Pallas, 1771 - Pallas Sailer (Nymphalidae) (Fig. 6f \& 6g)
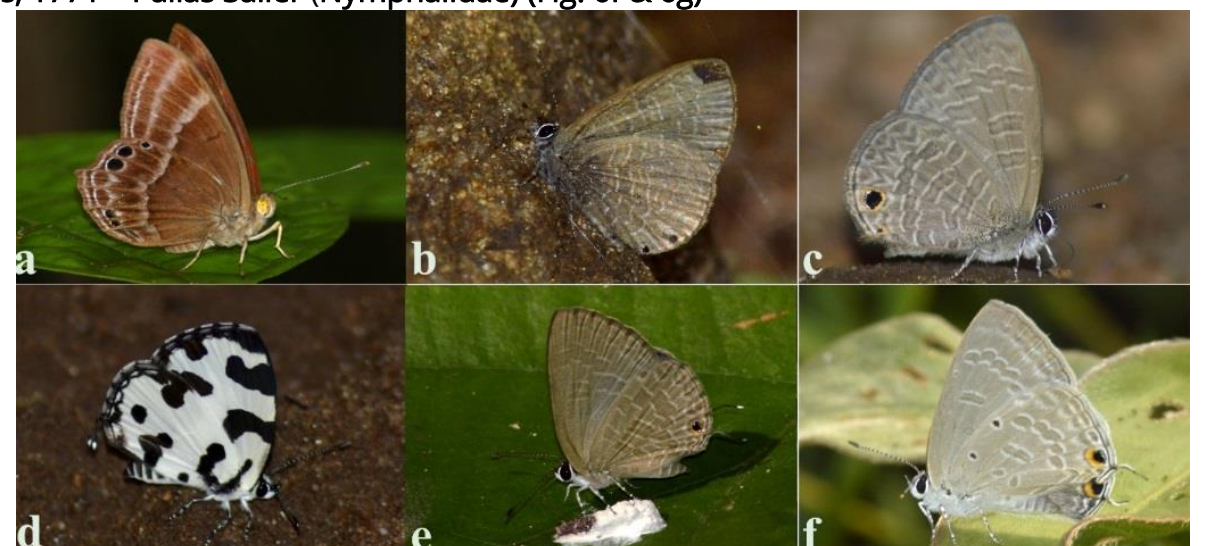

d
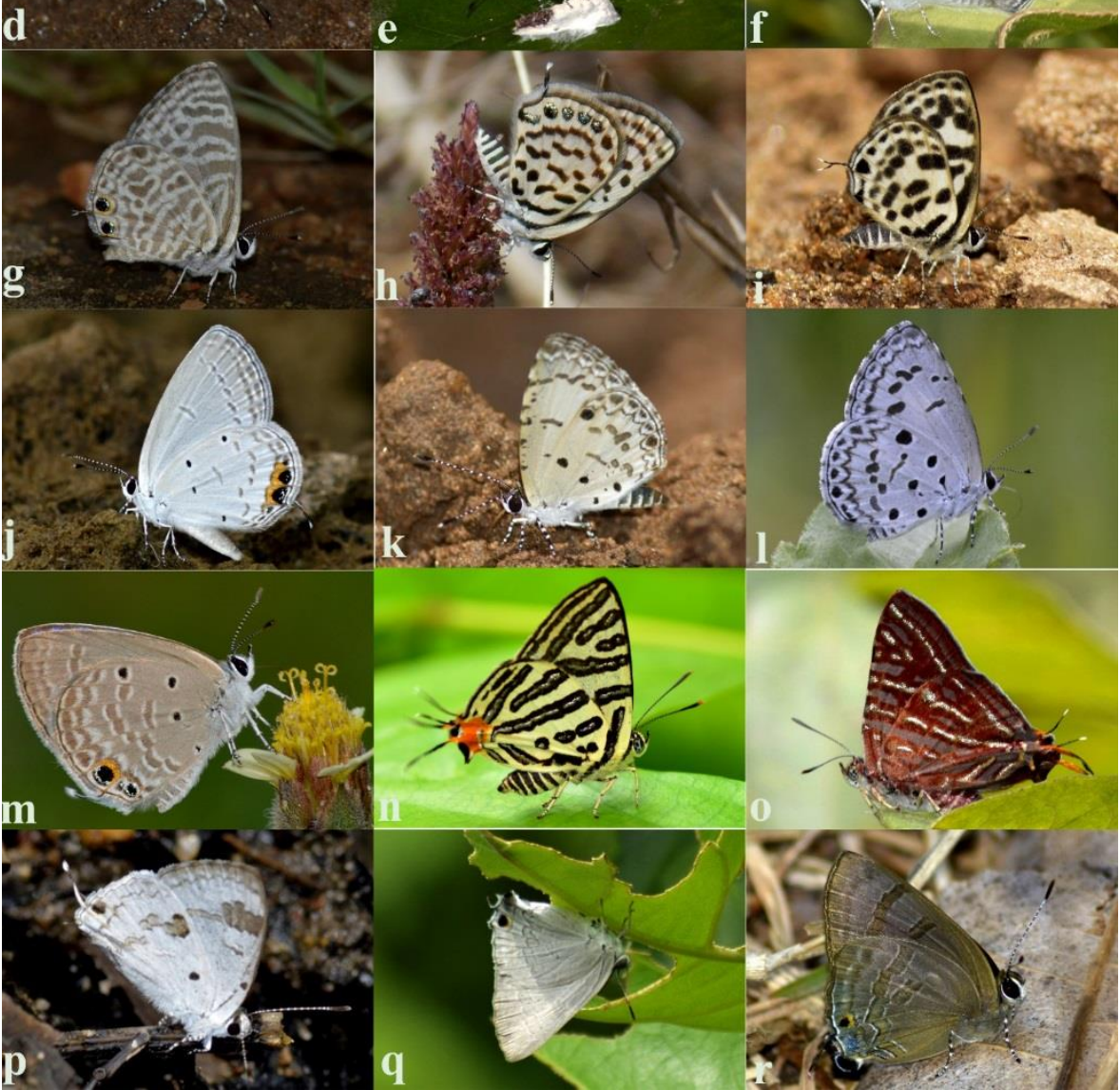

Fig 5. Photographs of Riodinidae and Lycaenidae butterflies: a - Abisara bifasciata; b - Petrelaea dana; c - Prosotas dubiosa; d - Caleta decidia; e - Jamides bochus, f - Catochrysops Strabo; g - Leptotes plinius, h - Tarucus sp; i - Tarucus ananda; j Everes lacturnus, k - Megisba malaya; I - Acytolepis puspa; m - Chilades parrhasius, n - Spindasis syama; o - Spindasis lohita; $\mathrm{p}$ - Chliaria othona; $\mathrm{q}$ - Virachola isocrates, $\mathrm{r}$ - Rapala varuna 


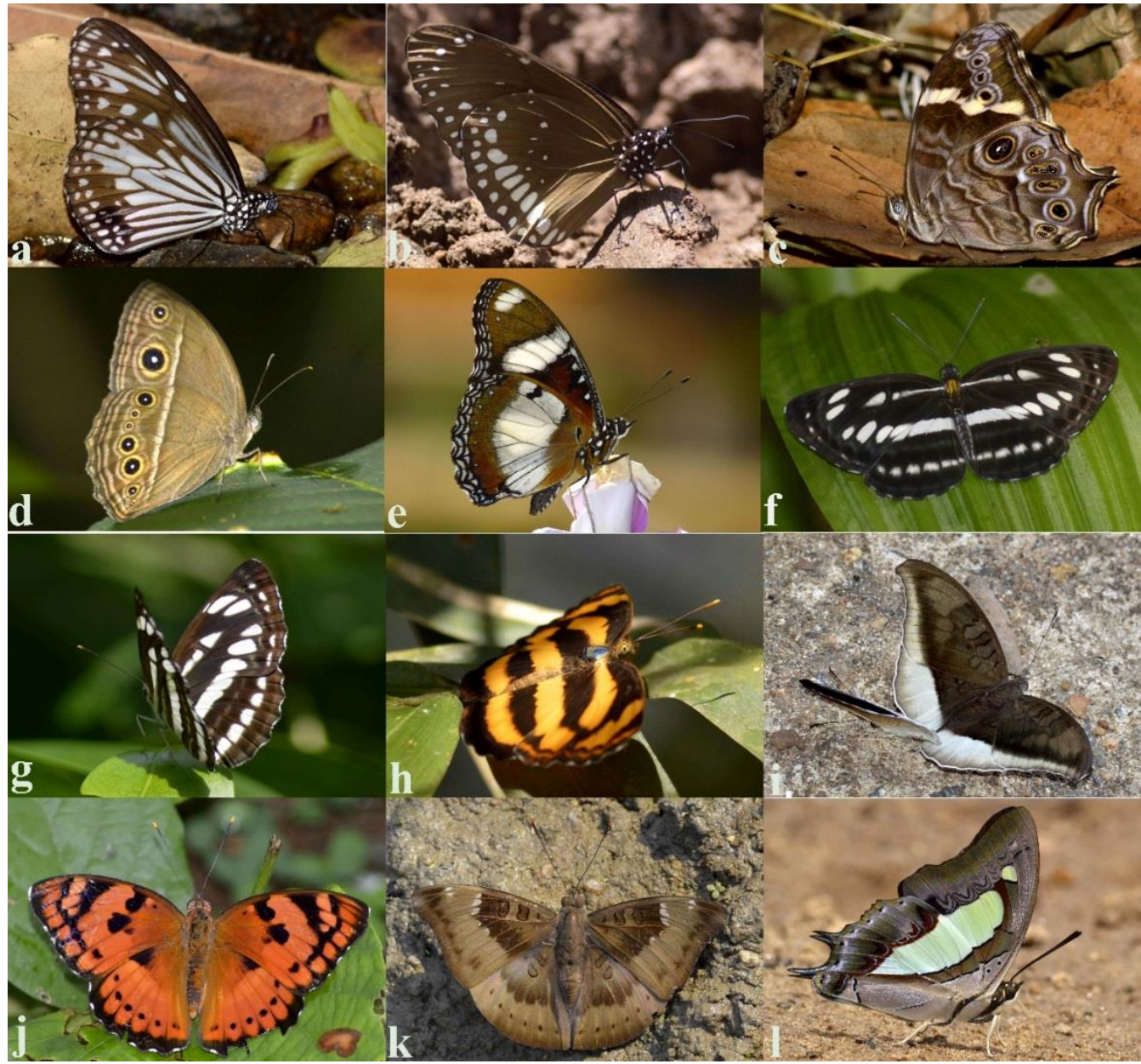

Fig. 6. Photographs of Nymphalidae butterflies: a - Parantica ag/ea; b - Euploea core; c - Lethe rohria; $\mathrm{d}$ - Mycalesis perseus, e - Hypolimnas misippus; f - Neptis Sappho (upper side); g - Neptis Sappho (under side); h - Pantoporia hordonia; i Tanaecia lepidea; j - Symphaedra nais, k - Euthalia aconthea; I - Polyura athamas

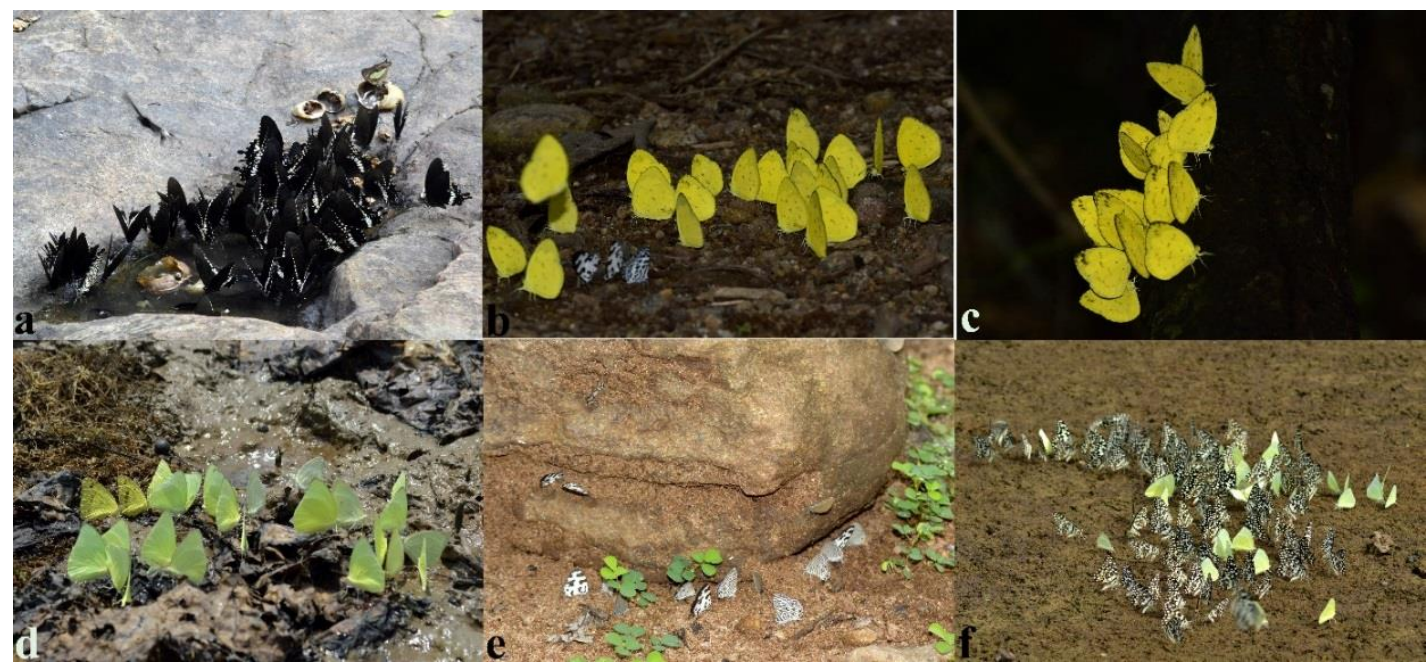

Fig. 7. Photographs of some butterfly congregation: a - Papilio polytes, Graphium doson, Papilio demoleus and Polyura athamas at Deobhuin Reserve Forest feeding on crab carcass and crab carcass mixed water; b - Eurema blanda, Eurema hecabe, Eurema andersonii, Caleta decidia and Castalius rosimon at Deobhin Reserve Forest puddling on forest trail; $c$ - E. andersonii feeding on tree trunk sap; d - Catopsilia pomona, Catopsilia pyranthe and Ixias marianne puddling on mud at Ansupa Lake; e - Prosotas dubiosa, Petrelaea dana, C. decidia, Leptotes plinius, Megisba malaya and Chilades pandava puddling at Balikiari Reserve Forest; $f$ - $P$. demoleus and $C$. pomona puddling on mud, near Sishupathra dam of Balikiari Beat

So far, this species has been known to distribute from Indian Himalaya and Northeastern India (Varshney \& Smetacek, 2015). Recently, the species has been recorded from Araku Valley and Maredumilli of Andhra Pradesh (Goswami et al., 2018). We spotted two individuals near Tersing on 03.09.2015 at 09:40. As Goswami et al. (2018) mentioned, this species can be differentiated from closely relative $N$. hylas, by "the veins in under-hindwing is not blackened and, in the forewing not blackened at least till cell" (Evans, 1932). Hence, our present record confirms its occurrence in Odisha for the first time. 


\section{Discussion}

The dominant occurrence of Lycenidae and Nymphalidae butterflies in the study area might be due to the availability of host plants and nectaring plants (Mimosaceae, Acanthaceae, Poaceae, Malvaceae, Fabaceae), as the habitat association of butterflies can be directly related to the availability of larval host plants, vegetation cover of herbs, shrubs and trees for nectaring (Thomas, 1995; Kunte, 2000).

Among the recorded 136 species, 113 species of butterflies were found from Balikiari Reserve Forest (Site 3), 105 species from Deobhuin Reserve Forest (Site 5), 88 from Ansupa Lake (Site 2), 84 from Tersing (Site 4) and 78 species from Dhabaleswar Island (Site 1). Result of high number of species in Balikiari and Deobhuin Reserve Forest compare to other sites like Tersing and Dhabaleswar Island, may be due to the longer period of surveys rather than true species richness. As site Tersing also endowed with pristine habitats, which can be suitable for many butterfly species those are yet to explore. Out of 136 species of butterflies, 14 species are legally protected under Indian Wildlife Protection Act, 1972 (see Table 2).

Table 2. Legally protected butterflies of Athgarh Forest Division under the Wildlife (Protection) Act, 1972 (WPA).

\begin{tabular}{lll}
\hline WPA Schedule & Family & Scientific Name \\
Schedule I & Papilionidae & Pachliopta hector(Linnaeus, 1758) \\
Schedule I & Lycaenidae & Chliaria othona (Hewitson, 1865) \\
Schedule II & Lycaenidae & Lachrysops chejus (Fabricius, 1798) \\
Schedule II & Lycaenidae & Rapala varuna Horsfield, 1829 \\
Schedule II & Lycaenidae & Spindasis lohita (Horsfield, [1829]) \\
Schedule II & Lycaenidae & Tanaecia lepidea Butler, 1868 \\
Schedule II & Nymphalidae & Hypolimnas misippus (Linnaeus, 1764) \\
Schedule II & Nymphalidae & Hasora vitta (Butler, 1870) \\
Schedule IV & Hesperiidae & Hyarotis adrastus (Stoll, [1780]) \\
Schedule IV & Hesperiidae & Baoris farri(Moore, 1878) \\
Schedule IV & Hesperiidae & Tarucus ananda (de Nicéville, [1884]) \\
Schedule IV & Lycaenidae & Euthalia lubentina Cramer, 1777 \\
Schedule IV & Nymphalidae & Euploea core (Cramer, [1780]) \\
Schedule IV & Nymphalidae &
\end{tabular}

In Athgarh Forest division various anthropogenic activities (e.g. wood cutting, grazing, logging, looping, herb collection etc.) by local villagers is a matter of concern, which leads to the destruction of suitable habitats of Butterfly fauna around Athgarh Forest Division. Butterflies are very sensitive insects. A little change in environmental condition and habitat alteration can influence their distribution and abundance (Wynter-Blyth, 1957). Therefore, habitat fragmentation, vegetation loss, grazing pressure, human settlement or any other damaging activities are mainly responsible for loss of diversity of both butterflies and plants in the study area must be regulated. Public awareness is also very important to conserve the suitable habitats of these ecologically important invertebrate organisms. However, the present study provides the baseline information of butterfly fauna of Athgarh Forest Division and enriches the butterfly checklist of Odisha. Further studies on Butterfly fauna is very necessary to understand the seasonal variation and population dynamics of butterflies in this precise geographical area.

\section{Acknowledgements}

Authors are grateful to Mr. Isaac Kehimkar (Director of INaturewatch Foundation, India), Mr. Monsoon Jyoti Gogoi (ScientistA, BNHS, India), and Mr. Gaurab Nandi Das for their valuable suggestions during identification. Authors are thankful to the Forest staffs of Athgarh Forest division for their kind assistance during survey period.

\section{References}

Annaandale, N., Drover, C. (1921). The Butterflies of Barkuda island. Record of the Indian Museum, $22(4), 349-375$. https://doi.org/10.5962/bhl.part.1476

Anonymous. 2019. Zographetus satwa de Nicéville, 1884 - Purple and Gold Flitter. Kunte, K., S. Sondhi, and P. Roy (Chief Editors). Butterflies of India, v. 2.63. Indian Foundation for Butterflies. Available from: http://www.ifoundbutterflies.org/sp/839/Zographetus-satwa

Boruah, B., Das, G.N., Payra, A., Gogoi, M.J., Dash, S.K., Tamuly, T., Sethy, J., Mishra, R.K. Rout, S.D. (2018). Assessment of Butterfly (Lepidoptera, Rhopalocera) Diversity in Manchabandha and Budhikhamari Reserve Forest, Mayurbhanj, Odisha, India. Asian Journal of Conservation Biology, 7 (1), 51-65.

Champion, H. G., Seth, S. K. (1968). Revised Forest Types of India. Manager of Publications, Govt of India, New Delhi.

Choudhury, K., H. Singha \& H.K. Sahu (2012). Swallowtail butterflies of Manas Biosphere Reserve of Northeast India - A pictorial Guide. Aaranyak, Guwahati, India.

Churi, P. 2019. Tarucus ananda (de Nicéville, [1884]) - Dark Pierrot. Kunte, K., S. Sondhi, and P. Roy (Chief Editors). Butterflies of India, v. 2.63. Indian Foundation for Butterflies. Available from: http://www.ifoundbutterflies.org/sp/664/Tarucus-ananda 
Crawford, W. M. (1921). Butterfly notes. J. Bombay nat. Hist. 80c., 28 (1), 292.

Das, S.K. Sahu, H.K. (2011). Preliminary study on Butterflies of Sunabeda Wildlife Sanctuary: A checklist with new records for Orissa, India. Indian Forester, 137(10), 1204-1206.

Evans, W. H. (1949). A catalogue of the Hesperiidae from Europe, Asia and Australia in the British Museum (N.H.). British Museum. https://doi.org/10.5962/bhl.title.105941

Evans, W.H. (1932). The Identification of Indian Butterflies. Second Edition, Bombay Natural History Society, Mumbai, India.

Goswami, R., Thorat, O., Aditya, V., Karimbumkara, S.N. (2018). A preliminary checklist of butterflies from the northern Eastern Ghats with notes on new and significant species records including three new reports for peninsular India. Journal of Threatened Taxa, 10(13), 12769-12791, doi: https://doi.org/10.11609/jott.3730.10.13.12769-12791

Gunathilagaraj, K., Perumal, T.N.A., Jayaram, K., Kumar, M.G. (1998). Some South Indian Butterflies: Field Guide. Project Lifescape, Indian Academy of Science, Bangalore.

Haribal, M. (1992). The Butterflies of Sikkim Himalaya. Sikkim Nature Conservation Foundation, Sikkim, India, 217.

Harisha, M.N., Hosetti B.B. (2013). Butterfly fauna of Daroji sloth bear sanctuary, Hospet, Bellary District, Karnataka, India. Journal of Research in Biology, 3(2), 840-846.

Kunte, K., Sondhi,S., Roy, P. (Chief Editors) (2019). Butterflies of India, v. 2.63. Indian Foundation for Butterflies. Available from: http://www.ifoundbutterflies.org

Kehimkar, I. (2008). The Book of Indian Butterflies. Bombay Natural History Society and Oxford University Press, Mumbai, India.

Kehimkar, I. (2016). Butterflies of India. Bombay Natural History Society, Mumbai.

Kunte, K. (2000). Butterflies of Peninsular India. Universities Press Limited, Hyderabad, India.

Kunte, K. (2019). Hasora vitta (Butler, 1870) - Plain Banded Awl. Kunte, K., S. Sondhi, and P. Roy (Chief Editors). Butterflies of India, v. 2.63. Indian Foundation for Butterflies.

Mandal, D.K., Maulik (1991). Insecta: Lepidoptera: Rhopalcera: Papilionidae, Papilioninae. A checklist. State Fauna Series-I, Fauna of Orissa, Part-I, Z.S.I. Calcutta, 235-238.

Mandal, D.K., Nandi, D.N. (1984). On Collection of Papilionidae from Orissa, India. Records of the Zoological Survey of India, 81, 355-368.

Mishra, S., Mahapatra, P.K., Sinha, S., Nayak, H.P., Mishra, A.K., Nair, M.V., Mahapatra, S.N., Panda, S. (2010). Butterflies Diversity of Nandankanan Wildlife Sanctuary, Odisha, India. e-planet, 8(1), 31-37.

Mohapatra, P.P., Sarkar, V., Mishra, A.K., Nair. M. V. (2012). A Field guide for beginners: Butterflies of Bonai, Odisha. Odisha Forestry Sector Development Project, Government of Odisha, Ministry of Environment and Forests, Bhubaneswar, Odisha, India.

Nair, M.V. (2007). Butterflies of Similipal Tiger Reserve: A preliminary study on species diversity, species composition and habitat preference, e-planet, 5(1), 76-81.

Nair, M.V. (2011). Three new butterfly records for peninsular India: Dusky Yellow-breasted Flat Gerosis phisara (Moore) (Hesperiidae), Common Gem Poritia hewitsoni Moore (Lycaenidae) and Great Nawab Polyura eudamippus (Doubleday) (Nymphalidae) from Similipal Hills, Odisha, India. Journal of Threatened Taxa, 3(3), 1624-1628. https://doi.org/10.11609/JoTT.02635.1624-8

Ogale, H., S. Sarang, A. Narkevar \& P. Churi. 2019. Hypolycaena othona Hewitson, [1865] - Orchid Tit. Kunte, K., S. Sondhi, and P. Roy (Chief Editors). Butterflies of India, v. 2.63. Indian Foundation for Butterflies. Available from: http://www.ifoundbutterflies.org/sp/509/Hypolycaena-othona

Palei, N.C., Rath, B.P. (2014). Butterflies Diversity of Sunabeda Wildlife Sanctuary, Odisha, India. Journal of Entomology and Zoological Studies, 2(2), 39-44.

Payra, A., Das, G. N., Boruah, B., Dash, S. K., Das, U. P., Sethy, J. (2016). Butterfly diversity in two selected fringe area of Similipal Biosphere Reserve, Odisha, India, with notes on some important sightings. Journal of Wildlife Research, 4(2), 17-25.

Sahu, H.K., Jena, J., Dutta, S.K., Rout, S.D. (2006). Common butterflies of Chahala Range of Similipal tiger Reserve, Odisha, India, Indian Forester, 32(10), 1363-1366.

Sethy, J., Jena, J. (2009). Notes on Butterflies of Gudgudia range of Simlipal Tiger Reseve, Orissa, India, Indian Forester, 135(10), 1442-1445.

Taylor, W. C., De Niceville, L. (1888). List of the Butterflies of Khordha in Orissa. Calcutta (Central Press Co.)

Thomas, J.A. (1995). The ecology and conservation of Maculineaarion and other European species of large blue butterfly. IN Ecology and Conservation of Butterflies (Ed. A.S. Pullin), Chapman and Hall, London, 180-210. https://doi.org/10.1007/978-94-011-1282$6 \underline{6} 13$

Tiple, A.D., Khurad, A.M. (2009). Butterfly Species Diversity, Habitats and Seasonal Distribution in and Around Nagpur City, Central India, World Journal of Zoology, 4(3), 153-162.

Varshney, R.K., Smetacek, P. (2015). A Synoptic Catalogue of the Butterflies of India. Butterfly Research Centre, Bhimtal.

Wynter-Blyth, M.A. (1957). Butterflies of the Indian Region. The Bombay Natural History Society, Bombay, India.

\section{Citation:}

Arajush Payra, Suraj K. Dash, Udit P. Das, Himanshu S. Palei, Arun K. Mishra (2019). Butterflies of Athgarh Forest Division, Odisha, Eastern India, with notes on some significant records. Acta Biologica Sibirica, 5 (3), $188-198$.

Submitted: 17.07.2019. Accepted: 23.09.2019.

cross ref http://dx.doi.org/10.14258/abs.v5.i3.6593

(C) 2019 by the authors. Submitted for possible open access publication under the terms and conditions of the

Creative Commons Attribution (CC BY) license (http://creativecommons.org/licenses/by/4.0/). 